\title{
The bulk motion of flat edge-on galaxies based on 2MASS photometry
}

\author{
Yu. N. Kudrya ${ }^{1}$, V. E. Karachentseva ${ }^{1}$, I. D. Karachentsev ${ }^{2}$, S. N. Mitronova ${ }^{2,5}$, T. H. Jarrett ${ }^{3}$, and W. K. Huchtmeier ${ }^{4}$ \\ 1 Astronomical Observatory of Kiev Taras Shevchenko National University, Observatorna str., 3, 04053 Kiev, Ukraine \\ 2 Special Astrophysical Observatory, Russian Academy of Sciences, N.Arkhyz, KChR, 369167, Russia \\ 3 Infrared Processing and Analysis Center, Mail Stop 100-22, California Institute of Technology, Jet Propulsion Laboratory, \\ Pasadena, CA 91125 \\ 4 Max-Planck - Institut für Radioastronomie, Auf dem Hügel 69, 53121, Bonn, Germany \\ 5 Isaac Newton Institute of Chile, SAO Branch, Russia
}

Received 11 February 2003/ Accepted 12 May 2003

\begin{abstract}
We report the results of applying the 2MASS Tully-Fisher (TF) relations to study galaxy bulk flows. For 1141 all-sky distributed flat RFGC galaxies we construct $J, H, K_{\mathrm{S}}$ TF relations and find that Kron $J_{\mathrm{fe}}$ magnitudes show the smallest dispersion on the TF diagram. For the sample of 971 RFGC galaxies with $V_{3 \mathrm{~K}}<18000 \mathrm{~km} \mathrm{~s}^{-1}$ we find a dispersion $\sigma_{\mathrm{TF}}=0.42^{\mathrm{m}}$ and an amplitude of bulk flow $V=199 \pm 61 \mathrm{~km} \mathrm{~s}^{-1}$, directed towards $l=301^{\circ} \pm 18^{\circ}, b=-2^{\circ} \pm 15^{\circ}$. Our determination of low-amplitude coherent flow is in good agreement with a set of recent data derived from EFAR, PSCz and SCI/SCII samples. The resultant two-dimensional smoothed peculiar velocity field traces well the large-scale density variations in the galaxy distributions. The regions of large positive peculiar velocities lie in the direction of the Great Attractor and Shapley concentration. A significant negative peculiar velocity is seen in the direction of Bootes and in the direction of the Local void. A small positive peculiar velocity (100-150 $\mathrm{km} \mathrm{s}^{-1}$ ) is seen towards the Pisces-Perseus supercluster, as well as the Hercules - Coma - Corona Borealis supercluster regions.
\end{abstract}

Key words. galaxies: spiral - galaxies: fundamental parameters

\section{Introduction}

Since the pioneering work by Aaronson et al. (1979, 1980, 1982), the infrared Tully-Fisher relation (IRTF) has been widely used in the study of galactic bulk flows on different scales. A rather complete review of the IRTF application is given in the thesis of Bamford (2002).

Cosmic flow investigations require both enormous and homogeneous samples. We can increase the amount of observational data by the sample combination procedure. For example, making the Mark III catalogue, Willick et al. (1997) compiled the infrared magnitudes and line widths from various sources and reduced them to a uniform system.

The appearance of the complete and homogeneous 2MASS survey Skrutskie et al. (1997) opens up new opportunities for cosmic flow study by IRTF. In our previous work (Karachentsev et al. 2002, hereafter Paper I) we identified the spiral edge-on galaxies from The Revised Flat Galaxy Catalogue, RFGC (Karachentsev et al. 1999) with the objects from The Extended Source Catalog, XSC (Cutri et al. 1998; Jarrett et al. 2000). Of the total number of 4236 RFGC galaxies, 2996 galaxies (e.g. 71\%) have been detected in the $J, H$,

Send offprint requests to: S. N. Mitronova,

e-mail: mit@luna.sao.ru
$K_{\mathrm{s}}$-bands. The RFGC catalogue was created using the material of the photographic sky surveys POSS-I and ESO/SERC. In Paper I we analyzed in detail the 2MASS-characteristics of flat galaxies entered in the XSC. To build the $B-, I-, J-, H-$, $K_{\mathrm{s}}$-TF relations, we used $B_{t}$-banditudes from RFGC, calculated from angular diameters, taking into account the galaxy surface brightness, as well as type, and other galaxy parameters; the total I-magnitudes from Mathewson \& Ford (1996) and Haynes et al. (1999); the isophotal $J-, H-, K_{\mathrm{s}}$-magnitudes measured in elliptical apertures at a level of $K_{\mathrm{s}}=20 \mathrm{mag} / \mathrm{arcsec}^{2}$. For a sample of 436 flat galaxies with this set of magnitudes and known radial velocities and HI line widths we obtained the slope of the linear TF regression, increasing from 4.9 in the $B$-band to 9.3 in the $K_{\mathrm{s}}$-band. The derived scatter on the TF diagram did not show a significant decrease from the blue to infrared band, and after excluding dwarf galaxies reached $\sim 0.6$ mag.

In the present work we study in the dipole approximation the bulk motion of flat galaxies from a homogeneous sample, the RFGC catalogue, using the $J_{-}, H_{-}, K_{\mathrm{s}}$-TF relations based on the 2MASS photometry data. We show that the scatter on the TF diagram can be diminished significantly by two factors: a cleaning of the initial sample and including additional photometric parameters in the simple TF relation. As a result, 
for 971 all-sky distributed flat galaxies the dipole solution is: $V=199 \mathrm{~km} \mathrm{~s}^{-1}, l=301^{\circ}, b=-2^{\circ}$. The smoothed peculiar velocity field repeats, as a whole, the large-scale distribution of flattened galaxies from the 2MASS Survey.

\section{The sample}

\subsection{The $H I$ line widths (the sample RFGC- $W_{50}$ ).}

In Paper I we presented the radial velocities of 1772 RFGC galaxies. However, not all of these 1772 objects have HI line width measurements. To compile the list of the RFGC galaxies with known estimates of $W_{50}$ (measured directly or calculated from rotation curves), we use the following sources:

a) The list of flat galaxies with known velocities and line widths (Karachentsev et al. 2000a). This compilation consists of several subsamples observed by Giovanelli et al. (1997a); Makarov et al. (1997a,b, 1999, 2001); Mathewson et al. (1992), Mathewson \& Ford (1996), and Matthews \& van Driel (2000). b) Our identifications of southern RFGC galaxies with the HIPASS survey sources (Karachentsev \& Smirnova 2002), and with "A Catalog of HI-Selected Galaxies from the South Celestial Cap Region of Sky" (Kilborn et al. 2002). c) The last version of LEDA database (Paturel et al. 1996). d) Unpublished data on the HI observations of RFGC galaxies at the Effelsberg and Nançay radio telescopes (Huchtmeier et al. 2003). The sources a), b), c), and d) contain, respectively, 78\%, 2\%, 11\%, and $9 \%$ of the whole sample. A total of 1653 sets of radial velocities, $V_{h}$, and line widths, $W_{50}$, were used in the initial list, including some multiple observations of certain galaxes. To check the "best" estimate among double and triple ones, we determine with a dipole approximation the distances $\mathrm{Hr}$, using the TF relation "linear diameter - line width" from Karachentsev et al. (2000b). The observed radial velocities were reduced to the cosmic microwave background $3 \mathrm{~K}$ system according to Kogut et al. (1993), and the observed line widths were corrected for cosmological broadening and turbulence following Tully \& Fouqué (1985). No corrections for inclination were made because the RFGC galaxies with their apparent optical axis ratio $a / b \geq 7$ are by definition very much inclined to the line of sight $\left(i \geq 82^{\circ}\right)$. The galaxy peculiar velocities were calculated as $V_{\mathrm{pec}}=V_{3 \mathrm{~K}}-H r$. Here and hereafter the inferred distance $H r$ is expressed in $\mathrm{km} \mathrm{s}^{-1}$. We retained in the RFGC- $\mathrm{W}_{50}$ sample only the galaxies from multiple observations whose peculiar velocities were minimal. In all the cases we took into account the galaxy morphology, possible confusion from a near neighbour, the signal/noise ratio etc. After excluding multiple measurements, 1617 RFGC galaxies with known radial velocities and line widths were entered in the RFGC-W 50 sample.

\subsection{The $2 M A S S$ photometry (the sample RFGC-2MASS)}

To compile this sample, we performed the cross-identification between RFGC and XSC catalogues. The initial file contains
3001 lines with 2MASS data. We used the following characteristics for the processing (Jarrett et al. 2000, 2003):

$r_{20}$ - major isophotal radius in arcsec, measured at the $20 \mathrm{mag} / \operatorname{arcsec}^{2}$ level in the $K_{\mathrm{s}}$ band via photometry in elliptical isophotes;

$r_{\mathrm{fe}}-$ fiducial elliptical Kron radius in arcseconds;

$r_{\text {ext }}$ - radius of the "total" aperture in arcseconds;

$J_{20 \mathrm{fe}}, H_{20 \mathrm{fe}}, K_{20 \mathrm{fe}}$ - isophotal fiducial elliptical-aperture magnitudes in corresponding bands measured at the $K_{\mathrm{s}}$-band fiducial $20 \mathrm{mag} / \operatorname{arcsec}^{2}$ isophotal radius;

$J_{\mathrm{fe}}, H_{\mathrm{fe}}, K_{\mathrm{fe}}-$ Kron fiducial elliptical-aperture magnitudes measured at the $K_{\mathrm{s}}$-band fiducial elliptical Kron radius;

$J_{\text {ext }}, H_{\text {ext }}, K_{\text {ext }}$ - integral "total" magnitudes as derived from the isophotal magnitudes $\left(J_{20}, H_{20}, K_{20}\right)$ and the extrapolation of the fit to the radial surface brightness distribution. The extrapolation $\left(r_{\text {ext }}\right)$ is carried out to roughly four times the disk scale length. (Details are given in Jarrett et al. 2003);

$J h l-J$-band "effective" surface brightness (at $J$ - band halflight "effective" radius);

$J c d e x-J$-band concentration index (3/4 vs. 1/4 light radius);

$s b a-$ axis ratio $(b / a)$ for the $J+H+K_{\mathrm{s}}$ combined image ("super" coadd).

A comparison of two lists, RFGC-2MASS and RFGC- $W_{50}$, gives 1215 common galaxies, including 68 galaxies with multiple 2MASS estimates. The selection of the best data among the duplicate ones is made by comparing the deviations from the simple linear TF relation:

$M=C_{1}+C_{2} \log W^{c}$,

where

$M=m-A-25-5 \log \left(V_{3 \mathrm{~K}} / H_{0}\right)$,

$A$ is extinction and $H_{0}=75 \mathrm{~km} \mathrm{~s}^{-1} \mathrm{Mpc}^{-1}$. It should be noted that the Eq. (2) is known to be incorrect at small distances since nearby galaxies around the Local Group do not need to have an additional $\sim 600 \mathrm{~km} \mathrm{~s}^{-1}$ correction subtracted from their apparent velocities to be placed in the proper Hubble flow. Altogether, 1141 galaxies were entered in the joint RFGC- $\mathrm{W}_{50}$ 2MASS sample after the excluding procedure. Some parameters of different observables are given in Table 1, where $\sigma$ is the standard deviation.

As known, the total and the Kron magnitudes are approximately $15-20 \%$ brighter than the isophotal magnitudes (see Figs. 11, 12 and 13 in http://spider.ipac. caltech. edu/staff/jarrett/papers/LGA /LGA_fig.htm).

\section{Construction of the optimal sample and calculation of the dipole parameters}

For each galaxy of our sample we have a set of the isophotal, Kron and the total magnitudes in the $J, H, K_{\mathrm{s}}$-bands. The verification of mutual correlations between all the magnitudes shows that they are well correlated (Table 2).

As an illustration of the tight mutual relationship between the 2MASS magnitudes, in Fig. 1 we give the regression of $K_{20 \text { fe }}$ on $H_{20 \text { fe }}$, consistent with the NIR colors of disk galaxies (Jarrett 2000; see also Fig. 20 in Jarrett et al. 2003). 
Table 1. Statistical characteristics of the flat galaxy sample.

\begin{tabular}{|c|c|c|c|c|}
\hline$N=1141$ & $\min$ & $\max$ & mean & $\sigma$ \\
\hline$J_{20 \mathrm{fe}}, \mathrm{mag}$ & 7.5 & 15.9 & 13.1 & 1.21 \\
\hline$H_{20 \text { fe }}$, mag & 6.6 & 15.4 & 12.3 & 1.23 \\
\hline$K_{20 \text { fe }}$, mag & 6.3 & 14.9 & 12.0 & 1.23 \\
\hline$J_{\mathrm{fe}}$, mag & 7.4 & 15.2 & 12.9 & 1.10 \\
\hline$H_{\text {fe }}$, mag & 6.6 & 14.7 & 12.1 & 1.13 \\
\hline$K_{\text {fe }}$, mag & 6.3 & 14.4 & 11.8 & 1.13 \\
\hline$J_{\text {ext }}$, mag & 7.4 & 15.9 & 12.9 & 1.13 \\
\hline$H_{\mathrm{ext}}$, mag & 6.5 & 15.3 & 12.1 & 1.16 \\
\hline$K_{\text {ext }}$, mag & 6.2 & 14.8 & 11.8 & 1.18 \\
\hline $\lg \left(r_{\mathrm{ext}}, \operatorname{arcsec}\right)$ & 0.82 & 2.36 & 1.57 & 0.22 \\
\hline $\lg \left(r_{\mathrm{fe}}, \operatorname{arcsec}\right)$ & 0.81 & 2.08 & 1.50 & 0.21 \\
\hline $\lg \left(r_{20 \mathrm{fe}}, \operatorname{arcsec}\right)$ & 0.70 & 2.04 & 1.36 & 0.25 \\
\hline$J h l, \mathrm{mag} / \operatorname{arcsec}^{2}$ & 16.8 & 20.6 & 19.0 & 0.68 \\
\hline Jcdex & 1.89 & 8.69 & 3.99 & 0.92 \\
\hline$s b a$ & 0.10 & 1.00 & 0.24 & 0.097 \\
\hline $\lg \left(W_{50}, \mathrm{~km} \mathrm{~s}^{-1}\right)$ & 1.36 & 2.89 & 2.50 & 0.163 \\
\hline$W_{50}, \mathrm{~km} \mathrm{~s}^{-1}$ & 23 & 782 & 335 & 112 \\
\hline $\lg \left(V_{3 \mathrm{~K}}, \mathrm{~km} \mathrm{~s}^{-1}\right)$ & 2.24 & 4.38 & 3.73 & 0.269 \\
\hline$V_{3 \mathrm{~K}}, \mathrm{~km} \mathrm{~s}^{-1}$ & 175 & 23758 & 6341 & 3327 \\
\hline
\end{tabular}

According to the data in Table 2, all three color bands seem to be of equal value.

To select between the nine different kinds of magnitudes, we built the TF relation (1) for each of them and calculated the characteristics of bulk motion in a dipole approximation. At this stage the apparent magnitudes were corrected for Galactic extinction as

$J^{\mathrm{c}}=J-0.207 \cdot A_{B}$,

$H^{\mathrm{c}}=H-0.132 \cdot A_{B}$,

$K^{\mathrm{c}}=K-0.084 \cdot A_{B}$,

where $A_{B}$ is extinction in the $B$-band according to Schlegel et al. (1988). Here we did not correct the magnitudes for internal galaxy extinction, because the internal extinction depends on the galaxy inclination, as well as the galaxy luminosity (Verheijen 2001), which is a priori unknown.

For each of the magnitudes we use constructed TF relations to calculate the characteristics of bulk motion in a dipole approximation. We adopt the simple linear model: $V_{\mathrm{pec}}=\boldsymbol{V} \boldsymbol{e}_{r}+\delta$, where $\boldsymbol{V}$ is the bulk velocity to the apex, $e_{r}$ is unit radial vector of galaxy direction. The value of $\Sigma \delta^{2}$ summing over the whole sample has been minimized by a least squares method.

The results of the calculations are given in Table 3 .

The columns of Table 3 denote:

(1) - 2MASS magnitude, by which the absolute magnitude in the TF relation was calculated;

(2) - dispersion on the TF diagram in mag;

(3) - slope $\mathrm{C}_{2}$ in formula (1) and its statistical significance according to the Fisher criterion in parentheses;

(4) - dispersion of peculiar velocities in $\mathrm{km} \mathrm{s}^{-1}$, which includes the error in the measured distances;

(5) - modulus of the bulk motion velocity and its error in $\mathrm{km} \mathrm{s}^{-1}$;

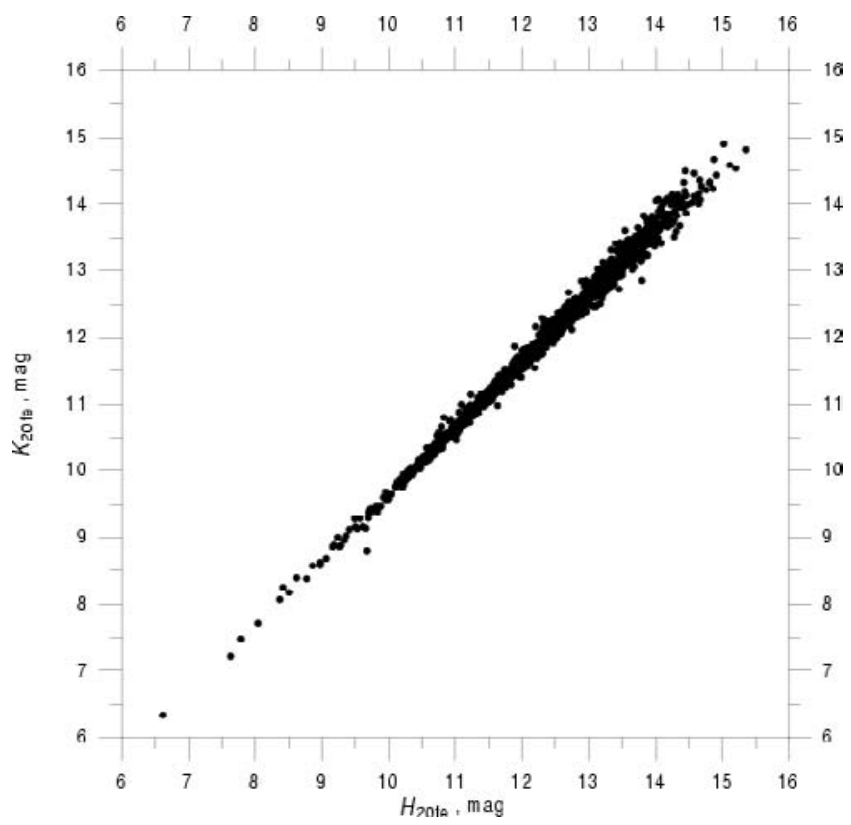

Fig. 1. The relationship between $H_{20 \mathrm{fe}}$ and $K_{20 \mathrm{fe}}$.

(6), (7) - galactic longitude and latitude of the apex and their errors in degrees;

(8) - significance of the vectorial dipole solution according to the Fisher criterion. (Note that for a confidence probability $95 \%$, the quantile of the Fisher distribution is equal to 2.6 for three degrees of freedom for the numerator and infinity for denominator.)

The errors in $V, l$, and $b$ were calculated by first calculating the diagonal elements $B_{V V}, B_{l l}$ and $B_{b b}$ of the covariance matrix $\mathbf{B}$ in the frame $\left\{\boldsymbol{e}_{V}, \boldsymbol{e}_{l}, \boldsymbol{e}_{b}\right\}$, and then $\Delta V=\left(B_{V V}\right)^{1 / 2}, \Delta l=$ $\arctan \left\{\left(B_{l l}\right)^{1 / 2} / V\right\}, \Delta l=\arctan \left\{\left(B_{b b}\right)^{1 / 2} / V\right\}$.

As seen from Table 3 , for every photometric magnitude the dispersion in the TF fit is too large, nearly 1.5 times as high as that presented in Paper I. We suggest therefore that the dipole parameters in Table 3 be considered only as preliminary. Due to a larger dispersion, the slopes of the TF relation are flatter than those obtained in Paper I.

It is also seen that the Kron magnitudes (especially $J_{\text {fe }}$ ) have a slightly lower dispersion in the TF diagram than isophotal as well as extended magnitudes. We built the diagrams of the residuals "isophotal minus Kron magnitude" depending on the absolute isophotal magnitude. For all three color bands in a wide range of absolute magnitudes $\left\{-16^{\mathrm{m}},-20^{\mathrm{m}}\right\}$ the Kron magnitudes are on average $\sim 0.4^{\mathrm{m}}$ brighter, thus recovering a significant fraction of the disk light that is lost in the background noise (see also Fig. 12 in Jarrett et al. 2003). This can reduce partially the known non-linearity of the $\mathrm{TF}$ relation at the lower luminous end.

We adopt $J_{\mathrm{fe}}$ as the basic apparent magnitude and trim the combined RFGC-W $\mathrm{W}_{50}-2 \mathrm{MASS}$ sample. The galaxies were omitted from the sample by following criteria:

1. $W_{50} \leq 100 \mathrm{~km} \mathrm{~s}^{-1}(N=13)$. These are bluish dwarf galaxies with large uncertainties in both the profile width and the IR magnitudes. 
Table 2. Some parameters of the mutually correlated linear dependence $y=k x+c$ between different magnitudes for the sample of 1141 galaxies.

\begin{tabular}{|c|c|c|c|c|c|c|c|}
\hline$x$ & $y$ & $\sigma_{\text {fwd }}$ & $\sigma_{\text {inv }}$ & $\sigma_{\text {orth }}$ & $r$ & $k$ & $c$ \\
\hline$J_{20 \mathrm{fe}}$ & $H_{20 \mathrm{fe}}$ & 0.119 & 0.117 & 0.083 & 0.9954 & $1.019 \pm 0.003$ & $-1.042 \pm 0.039$ \\
\hline$J_{20 \mathrm{fe}}$ & $K_{20 \mathrm{fe}}$ & 0.162 & 0.159 & 0.114 & 0.9913 & $1.019 \pm 0.004$ & $-1.404 \pm 0.053$ \\
\hline$H_{20 \mathrm{fe}}$ & $K_{20 \mathrm{fe}}$ & 0.114 & 0.114 & 0.080 & 0.9958 & $1.000 \pm 0.003$ & $-0.361 \pm 0.034$ \\
\hline$J_{\mathrm{fe}}$ & $H_{\mathrm{fe}}$ & 0.131 & 0.127 & 0.091 & 0.9933 & $1.026 \pm 0.004$ & $-1.107 \pm 0.046$ \\
\hline$J_{\mathrm{fe}}$ & $K_{\mathrm{fe}}$ & 0.173 & 0.168 & 0.121 & 0.9882 & $1.026 \pm 0.005$ & $-1.460 \pm 0.061$ \\
\hline$H_{\mathrm{fe}}$ & $K_{\mathrm{fe}}$ & 0.131 & 0.131 & 0.093 & 0.9932 & $1.000 \pm 0.003$ & $-0.352 \pm 0.042$ \\
\hline$J_{\text {ext }}$ & $H_{\text {ext }}$ & 197 & 0.193 & 0.138 & 354 & 005 & .068 \\
\hline$J_{\text {ext }}$ & $K_{\text {ext }}$ & 0.223 & 0.214 & 0.155 & 0.9820 & $1.043 \pm 0.006$ & .077 \\
\hline$H_{\mathrm{ext}}$ & $K_{\text {ext }}$ & 0.190 & 0.187 & 0.133 & 0.9870 & $1.018 \pm 0.005$ & .060 \\
\hline$J_{20 \mathrm{fe}}$ & $J_{\mathrm{fe}}$ & 0.146 & 0.161 & 0.108 & 0.9912 & $0.905 \pm$ & .047 \\
\hline$H_{20 \mathrm{fe}}$ & $H_{\mathrm{fe}}$ & 0.151 & 0.165 & 0.111 & 0.9910 & $0.911 \pm 0.004$ & $+0.898 \pm 0.045$ \\
\hline$K_{20 \mathrm{fe}}$ & $K_{\mathrm{fe}}$ & 0.143 & 0.157 & 0.106 & 0.9919 & $0.911 \pm 0.003$ & $+0.874 \pm 0.042$ \\
\hline$J_{\mathrm{fe}}$ & $J_{\text {ext }}$ & 0.165 & 0.160 & 0.115 & 0.9894 & $1.030 \pm 0.004$ & $4 \pm 0.058$ \\
\hline$H_{\mathrm{fe}}$ & $H_{\mathrm{ext}}$ & 0.177 & 0.172 & 0.124 & 0.9883 & $1.030 \pm 0.005$ & $-0.373 \pm 0.057$ \\
\hline$K_{\mathrm{fe}}$ & $K_{\text {ext }}$ & 0.150 & 0.144 & 0.104 & 0.9919 & $1.048 \pm 0.004$ & $-0.544 \pm 0.047$ \\
\hline
\end{tabular}

Table 3. Parameters of the TF relation (1) and the dipole characteristics for 1141 flat galaxies.

\begin{tabular}{|l|c|c|c|c|c|c|c|}
\hline \hline & $\sigma_{\mathrm{TF}}$ & $C_{2}$ & $\sigma_{V}$ & $V$ & $l$ & $b$ & $F$ \\
\hline$J_{\mathrm{fe}}$ & 0.855 & $-6.24 \pm 0.15(1653)$ & 1940 & $416 \pm 106$ & $284 \pm 15$ & $-21 \pm 12$ & 5.2 \\
$J_{20 \mathrm{fe}}$ & 0.940 & $-6.81 \pm 0.17(1630)$ & 2177 & $568 \pm 118$ & $292 \pm 12$ & $-24 \pm 10$ & 7.8 \\
$J_{\text {ext }}$ & 0.911 & $-6.50 \pm 0.16(1581)$ & 2076 & $437 \pm 113$ & $287 \pm 15$ & $-17 \pm 12$ & 5.0 \\
\hline$H_{\mathrm{fe}}$ & 0.887 & $-6.50 \pm 0.16(1668)$ & 2033 & $448 \pm 111$ & $287 \pm 15$ & $-23 \pm 12$ & 5.5 \\
$H_{20 \mathrm{fe}}$ & 0.972 & $-7.05 \pm 0.17(1635)$ & 2267 & $565 \pm 122$ & $293 \pm 13$ & $-24 \pm 10$ & 7.2 \\
$H_{\text {ext }}$ & 0.947 & $-6.78 \pm 0.17(1592)$ & 2183 & $443 \pm 119$ & $288 \pm 16$ & $-24 \pm 13$ & 4.7 \\
\hline$K_{\text {fe }}$ & 0.907 & $-6.68 \pm 0.16(1686)$ & 2062 & $340 \pm 113$ & $285 \pm 19$ & $-22 \pm 15$ & 3.1 \\
$K_{20 \mathrm{fe}}$ & 0.990 & $-7.21 \pm 0.18(1647)$ & 2295 & $475 \pm 124$ & $292 \pm 15$ & $-23 \pm 12$ & 4.9 \\
$K_{\text {ext }}$ & 0.966 & $-6.99 \pm 0.17(1625)$ & 2226 & $378 \pm 121$ & $290 \pm 19$ & $-22 \pm 15$ & 3.3 \\
\hline
\end{tabular}

2. The deviation from the TF relation is more than $3 \sigma$ $(N=63)$.

3. $\left|V_{\text {pec }}\right| \geq 3000 \mathrm{~km} \mathrm{~s}^{-1}(N=119)$. We assume that such large peculiar velocity values are due to non-physical reasons.

We also eliminate 6 galaxies with $V_{3 \mathrm{~K}} \geq 18000 \mathrm{~km} \mathrm{~s}^{-1}$ and (symmetrically) 10 galaxies with $\mathrm{Hr} \geq 18000 \mathrm{~km} \mathrm{~s}^{-1}$ to diminish the incompleteness of the sample at large distances. The process of elimination converged after 5 steps; altogether, taking into account the cross-sections, we excluded 170 galaxies.

Excluding the dwarf galaxies with smallest velocity widths means simultaneously eliminating the nearest galaxies. For the new sample of 971 galaxies the minimal value of $V_{3 \mathrm{~K}}$ increases to $494 \mathrm{~km} \mathrm{~s}^{-1}$ in comparison with $175 \mathrm{~km} \mathrm{~s}^{-1}$ for the noncleaned sample. Note that from the total number of 971 galaxies, about $87 \%$ galaxies have $V_{3 \mathrm{~K}}>3000 \mathrm{~km} \mathrm{~s}^{-1}$, i.e. lie outside the Local Supercluster. Thus, we believe the contribution of nearby galaxies to the bulk motion parameters (when the CMB frame is used) is not significant.

The TF relation for the new sample of 971 galaxies $M\left(J_{\mathrm{fe}}\right)$ vs. $\log W^{c}$, is given in Fig. 2. It has a slope $C_{2}=-8.1$ and a dispersion $\sigma_{\mathrm{TF}}=0.430$. The bulk motion parameters obtained from this relation are:

$\sigma_{V}=1062 \mathrm{~km} \mathrm{~s}^{-1}, V=323 \mathrm{~km} \mathrm{~s}^{-1}$,

$l=293^{\circ}, b=-15^{\circ}$.

As is seen in Fig. 2, eliminating $15 \%$ of the initial sample using the "clean" criteria given above greatly reduces the dispersion in the TF relation.

Next, for the sample of $N=971$ we consider a sevenparameter generalized TF relation, in which photometric characteristics and color index are entered:

$$
\begin{aligned}
M\left(J_{\mathrm{fe}}\right)= & C_{1}+C_{2} \cdot \lg \left(W^{c}\right)+C_{3} \cdot j h l+C_{4} \cdot j c d e x \\
& +C_{5} \cdot\left(J_{\mathrm{fe}}-K_{\mathrm{fe}}\right)+C_{6} \cdot s b a+C_{7} \cdot \lg \left(r_{\mathrm{ext}}\right) .
\end{aligned}
$$




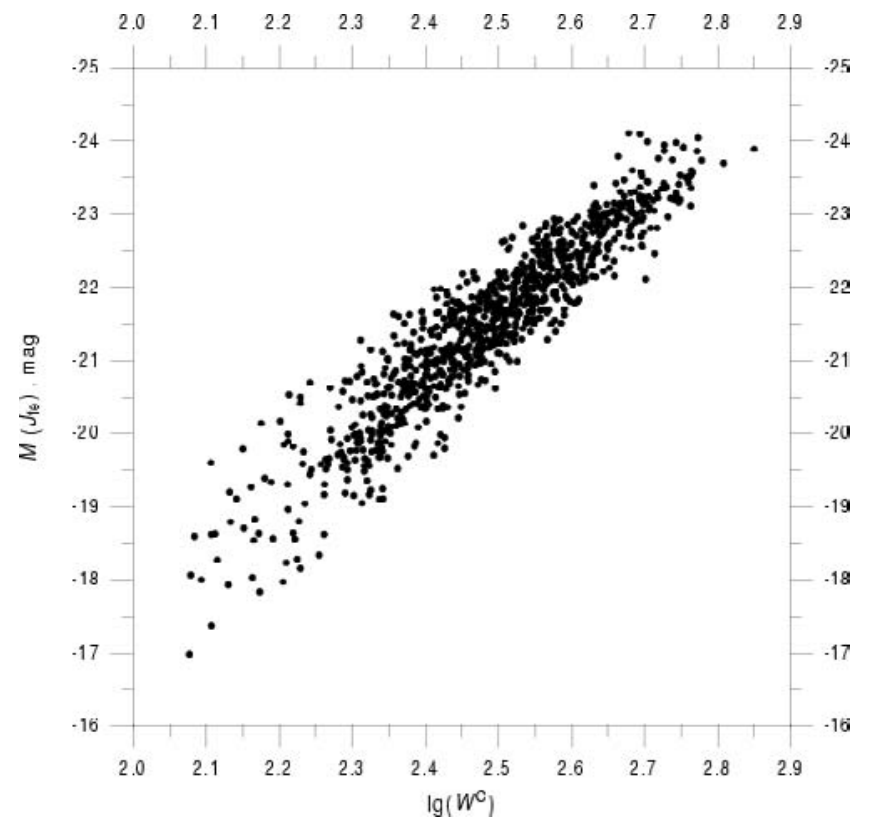

Fig. 2. The Tully-Fisher relation for 971 flat edge-on galaxies.

For the seven-parameter TF relation (4) the solutions are: the slope $C_{2}=-7.6, \sigma_{\mathrm{TF}}=0.423, \sigma_{V}=1044 \mathrm{~km} \mathrm{~s}^{-1}, V=$ $187 \mathrm{~km} \mathrm{~s}^{-1}, l=300^{\circ}, b=-1^{\circ}$. The $J h l,\left(J_{\mathrm{fe}}-K_{\mathrm{fe}}\right)$ and $s b a$ terms are statistically insignificant. In this case, only four terms remain in the new generalized TF relation:

$M\left(J_{\mathrm{fe}}\right)=C_{1}+C_{2} \cdot \lg \left(W^{c}\right)+C_{3} \cdot j c d e x+C_{4} \cdot \lg \left(r_{\mathrm{ext}}\right)$.

The new parameters are practically the same as for (4): zeropoint $C_{1}=-1.6$, slope $C_{2}=-7.6, C_{3}=-0.046, C_{4}=-0.44$, $\sigma_{\mathrm{TF}}=0.422, \sigma_{V}=1045 \mathrm{~km} \mathrm{~s}^{-1}, V=199 \mathrm{~km} \mathrm{~s}^{-1}, l=301^{\circ}$, $b=-2^{\circ}$.

The significance of the jcdex term $(F=4.5)$ means that the TF relations depend on the Hubble type, namely the central bulge to disk distribution. Statistical insignificance of the $s b a$ term $(F=0.3)$ indicates that internal extinction in the infrared does not correlate, surprisingly, with the infrared axial ratio. The influence of the surface brightness term, $j h l$, in the regression (4) falls, but its role is partially evident via the $\lg \left(r_{\text {ext }}\right)$ term $(F=17.5)$. In Fig. 3 we give distances $\mathrm{Hr}$ derived from the TF relation (5) depending on radial velocities $V_{3 \mathrm{~K}}$ for the sample $N=971$.

We also built analogous (seven- and four-parametric) TF relations based on the other eight 2MASS photometric measures. They demonstrate the same tendencies: the terms $J h l,\left(J_{\mathrm{fe}}-K_{\mathrm{fe}}\right)$ and $s b a$ also are insignificant and the dipole bulk motion parameters are near to the ones mentioned above.

The results of calculations with nine TF relations (5) for the sample of galaxies with $V_{3 \mathrm{~K}}<18000 \mathrm{~km} \mathrm{~s}^{-1}$ and $\mathrm{Hr}<$ $18000 \mathrm{~km} \mathrm{~s}^{-1}$ are collected in Table 4. The designations are the same as in Table 3.

We also constructed the second sample with the same restrictions as for the sample $N=971$, but with $V_{3 \mathrm{~K}}<$ $12000 \mathrm{~km} \mathrm{~s}^{-1}$ and $H r<12000 \mathrm{~km} \mathrm{~s}^{-1}$, to diminish the influence of data incompleteness at large distances. In Table 5 we show the same data as in Table 4 but for the sample of galaxies

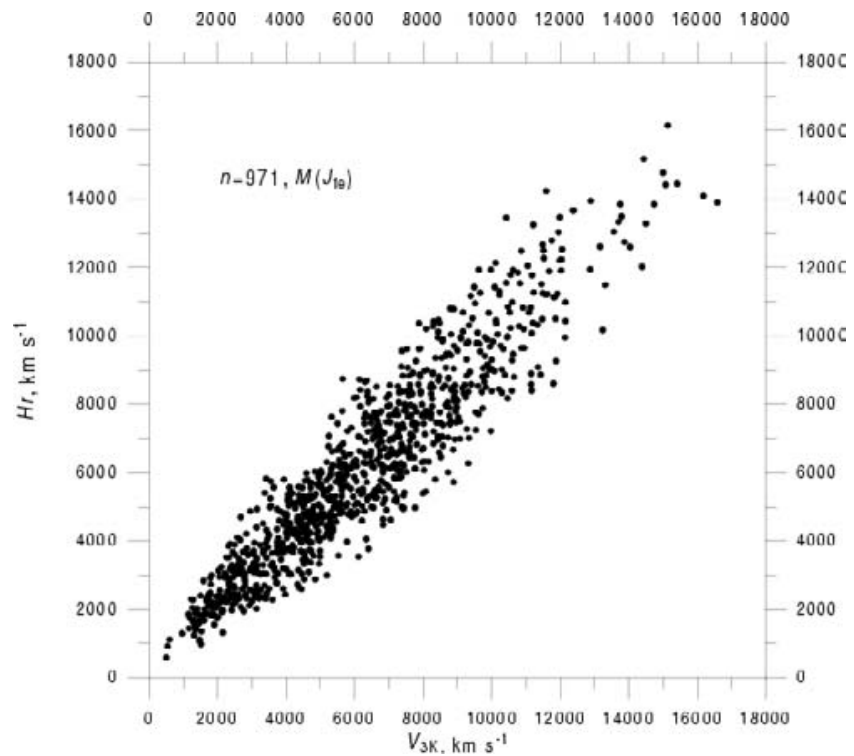

Fig. 3. The relationship between the derived distances $\mathrm{Hr}$ and radial velocities $V_{3 \mathrm{~K}}$.

with $V_{3 \mathrm{~K}}<12000 \mathrm{~km} \mathrm{~s}^{-1}$ and $H r<12000 \mathrm{~km} \mathrm{~s}^{-1}$. The designations are the same as in Table 3 . The elimination of 50 remote galaxies does not change the dipole parameters much nor the TF parameters $C_{i}$.

Comparing the data in Tables 3-5 allows us to conclude:

1) The cleaning of the sample diminishes significantly the dispersion $\sigma_{\mathrm{TF}}(\sim 2$ times $)$ and the bulk motion velocity modulus (about 1.5-2.5 times).

2) In all procedures (the cleaning of the sample, the use of regression with other magnitudes) the galactic longitude of the apex changes little, within a $20^{\circ}$ range.

3) The cleaning of the sample moves the apex to the galactic equator.

\section{Peculiar velocity field}

Based on a four-parametric regression (5) and derived peculiar velocities, we built the peculiar velocity field of the RFGC galaxies for the sample $N=921$. In Fig. 4 we present a smoothed peculiar velocity field for them in supergalactic coordinates SGL, SGB. The solid line indicates the "Zone of Avoidance" of \pm 10 degrees around the Galactic equator, where effects from reddening, stellar contamination and incompleteness are significant. The galaxies with $V_{\text {pec }}>0$ are designated by open circles, and the galaxies with $V_{\text {pec }}<0$ with filled circles. The smoothing was done with a Gaussian filter of $20^{\circ}$ window. The color scale at the bottom of Fig. 4 shows a range of average peculiar velocities from $-200 \mathrm{~km} \mathrm{~s}^{-1}$ to $+500 \mathrm{~km} \mathrm{~s}^{-1}$.

In Fig. 5 we give the sky distribution in SGL, SGB of 22361 galaxies from the 2MASS survey selected by the infrared axial ratio criterion $s b a<0.3$. On the bottom right, the positions of the Great Attractor (GA), the Pisces-Perseus supercluster (PP), the Shapley concentration (Sh), as well as the Local Void (LV) and the Bootes Void (BV) are marked. Comparison of Figs. 4 and 5 shows that the regions with positive and negative smoothed velocities reveal, as a whole, the 
Table 4. Parameters of TF regressions (5) for the 2MASS magnitudes and the dipole characteristics for 971 flat galaxies.

\begin{tabular}{|l|c|c|c|c|c|c|c|}
\hline \hline & $\sigma_{\mathrm{TF}}$ & $C_{2}$ & $\sigma_{V}$ & $V$ & $l$ & $b$ & $F$ \\
\hline$J_{\mathrm{fe}}$ & 0.422 & $-7.64 \pm 0.13(3558)$ & 1045 & $199 \pm 61$ & $301 \pm 18$ & $-2 \pm 15$ & 3.5 \\
$J_{20 \mathrm{fe}}$ & 0.457 & $-8.21 \pm 0.14(3505)$ & 1144 & $248 \pm 67$ & $306 \pm 16$ & $-5 \pm 13$ & 4.6 \\
$J_{\text {ext }}$ & 0.464 & $-7.85 \pm 0.14(3111)$ & 1153 & $215 \pm 67$ & $302 \pm 18$ & $+7 \pm 15$ & 3.5 \\
\hline$H_{\text {fe }}$ & 0.437 & $-7.86 \pm 0.13(3509)$ & 1078 & $209 \pm 64$ & $294 \pm 18$ & $-9 \pm 16$ & 3.6 \\
$H_{20 \mathrm{fe}}$ & 0.471 & $-8.45 \pm 0.14(3490)$ & 1167 & $239 \pm 69$ & $304 \pm 17$ & $-7 \pm 16$ & 4.0 \\
$H_{\text {ext }}$ & 0.485 & $-8.15 \pm 0.15(3067)$ & 1194 & $198 \pm 70$ & $288 \pm 20$ & $0 \pm 17$ & 2.7 \\
\hline$K_{\text {fe }}$ & 0.445 & $-8.14 \pm 0.13(3635)$ & 1116 & $153 \pm 66$ & $294 \pm 24$ & $-1 \pm 20$ & 1.8 \\
$K_{20 \mathrm{fe}}$ & 0.483 & $-8.72 \pm 0.15(3552)$ & 1209 & $201 \pm 71$ & $300 \pm 20$ & $-3 \pm 18$ & 2.7 \\
$K_{\text {ext }}$ & 0.480 & $-8.34 \pm 0.15(3289)$ & 1204 & $138 \pm 69$ & $301 \pm 28$ & $+12 \pm 24$ & 1.4 \\
\hline
\end{tabular}

Table 5. Parameters of TF regressions (5) for the 2MASS magnitudes and the dipole characteristics for 921 flat galaxies.

\begin{tabular}{|l|c|c|c|c|c|c|c|}
\hline \hline & $\sigma_{\mathrm{TF}}$ & $C_{2}$ & $\sigma_{V}$ & $V$ & $l$ & $b$ & $F$ \\
\hline$J_{\mathrm{fe}}$ & 0.432 & $-7.64 \pm 0.14(3033)$ & 1023 & $226 \pm 62$ & $295 \pm 16$ & $-2 \pm 13$ & 4.5 \\
$J_{20 \mathrm{fe}}$ & 0.466 & $-8.20 \pm 0.15(2993)$ & 1111 & $271 \pm 67$ & $301 \pm 15$ & $-5 \pm 12$ & 5.4 \\
$J_{\text {ext }}$ & 0.473 & $-7.83 \pm 0.15(2657)$ & 1113 & $254 \pm 66$ & $299 \pm 16$ & $+6 \pm 13$ & 5.0 \\
\hline$H_{\text {fe }}$ & 0.446 & $-7.84 \pm 0.14(2995)$ & 1045 & $242 \pm 64$ & $289 \pm 15$ & $-12 \pm 14$ & 4.7 \\
$H_{20 \mathrm{fe}}$ & 0.481 & $-8.42 \pm 0.15(2978)$ & 1129 & $263 \pm 69$ & $298 \pm 15$ & $-9 \pm 14$ & 4.9 \\
$H_{\text {ext }}$ & 0.495 & $-8.11 \pm 0.16(2601)$ & 1156 & $243 \pm 71$ & $289 \pm 17$ & $-4 \pm 14$ & 4.0 \\
\hline$K_{\mathrm{fe}}$ & 0.453 & $-8.10 \pm 0.15(3101)$ & 1067 & $195 \pm 65$ & $288 \pm 19$ & $-4 \pm 16$ & 3.0 \\
$K_{20 \mathrm{fe}}$ & 0.491 & $-8.68 \pm 0.16(3033)$ & 1151 & $240 \pm 70$ & $295 \pm 17$ & $-5 \pm 15$ & 3.9 \\
$K_{\text {ext }}$ & 0.487 & $-8.28 \pm 0.16(2802)$ & 1141 & $183 \pm 68$ & $295 \pm 21$ & $+6 \pm 18$ & 2.5 \\
\hline
\end{tabular}

galaxy overdense and underdense regions. The regions with maximal positive $V_{\text {pec }}$ lie in the direction of the GA and the Shapely Concentration. Note that the obscuring effects on the Milky Way are also at a maximum towards these regions (e.g., Abell 3627 of the GA region is well within the "Zone of Avoidance"), rendering large regions of the sky incomplete in the 2MASS XSC and the RFGC. Significant negative $V_{\text {pec }}$ are seen towards the voids, and low positive velocities $(\sim 100-$ $150 \mathrm{~km} \mathrm{~s}^{-1}$ ) are seen in the PP and Hercules - Coma - Corona Borealis superclusters.

\section{Comparison with other data}

To estimate the amount and quality of the observational material used to determine the bulk motion parameters, we introduce the value $G$, the sample goodness

$G=(N / 100)^{1 / 2} / \sigma_{\mathrm{TF}}$.

Here $N$ is the number of galaxies under study and $\sigma_{\mathrm{TF}}$ is their dispersion on the TF diagram. From the papers of different authors, we took a sample of spiral galaxies lying mainly in sparse regions. In Table 6 we give: (1) designation of the sample, (2) color band used, (3) number of galaxies in the sample, (4) the dispersion on the TF diagram, (5) the sample goodness, (6) source of data. The upper four lines note the samples included in the Mark III catalog with their respective name. The data for them are taken from Tables 1 and 3 in Willick et al. (1997). The next three samples are interesting because of their relative large depth $\left(c z \sim 10000 \mathrm{~km} \mathrm{~s}^{-1}\right)$. The sample of Bamford (2002) was selected from the SCI sample of Giovanelli et al. (1997b). From $782 \mathrm{~S}$ galaxies placed in the clusters or groups, Bamford selected 153 galaxies with available 2MASS photometry. Other than Bamford's data, we have not yet encountered published TF results using 2MASS.

In the last line we give the results obtained in this paper. As seen, the data goodness varies over a rather wide range, and the larger samples have, naturally, higher values of $G$.

Note that different authors use ways of creating their "pure" samples. In the case of our sample, we have not yet made a detailed analysis of the observable errors, nor reduced the HI line measurements to a common system, nor analyzed the Malmquist bias. We excluded the outlying galaxies only statistically because of their greater than $3 \sigma$ deviations from the TF fit and large calculated $V_{\text {pec }}$. Nevertheless, our data are of high accurate $(G=7.4$ in comparison with the median 4.8 for the samples in Table 6). As the minimal scatter on the TF diagram has a finite intrinsic value $(0.25-0.30 \mathrm{mag})$, the improvement of the data is undoubtedly associated with the increased sample size.

Let us compare briefly our results with the recent literature data. The most representative sample in Table 6, the Mark III catalog, yields the bulk motion $V=370 \pm 110 \mathrm{~km} \mathrm{~s}^{-1}$ towards $l=305^{\circ}, b=+14^{\circ}$ in the region $\mathrm{Hr}<5000 \mathrm{~km} \mathrm{~s}^{-1}$ (Dekel et al. 1999). Our dipole solution is consistent with the Mark III data. Zaroubi (2002) presents a review of the latest results of galaxy bulk motion measurements accomplished by different authors based on various observational measurements. 

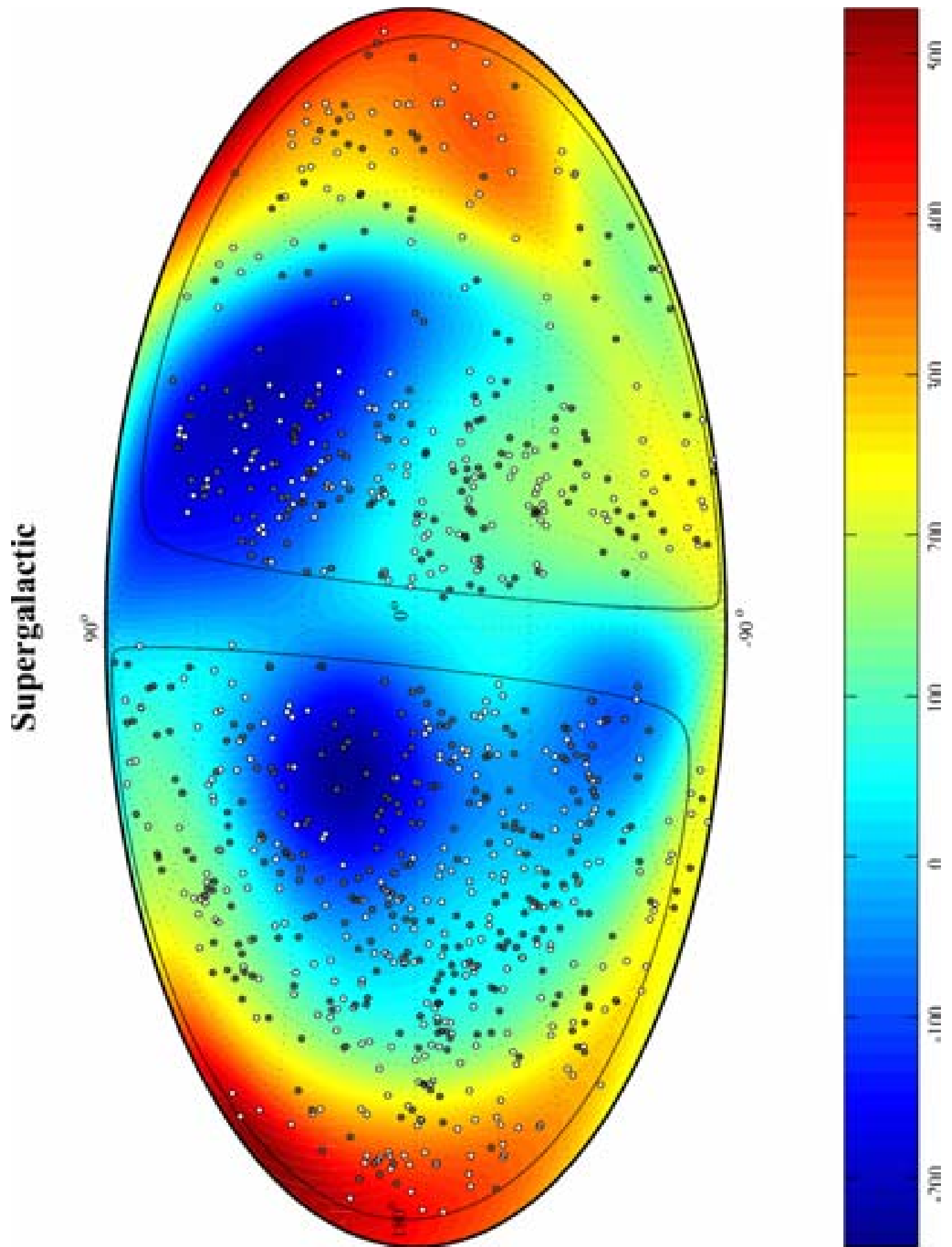

Fig. 4. A smoothed peculiar velocity field for 921 RFGC galaxies. The galaxies with $V_{\text {pec }}>0$ and $V_{\text {pec }}<0$ are designated as open and filled circles, respectively. The "Zone of Avoidance" is indicated by a solid line.

We compare our result with other dipole determinations using Fig. 1 and Table 1 from Zaroubi (2002). Our RFGC sample can be considered as relatively distant (its depth reaches $\left.18000 \mathrm{~km} \mathrm{~s}^{-1}\right)$. At comparable distances $\left(\sim 100-150 \mathrm{Mpc}^{-1}\right)$ according to the Zaroubi compilation, the bulk motion parameters were determined for the samples of distant elliptical galaxies, EFAR (Colless et al. 2001), distant Abell clusters, LP10 (Willick 1999), point sources from IRAS $z$-catalog (PSCz)
(Saunders et al. 2000; Branchini et al. 2000), spiral galaxies in clusters and superclusters (Dale et al. 1999), and distant clusters, LP (Lauer \& Postman 1994).

The low amplitude of bulk motion, $V=199 \pm 62 \mathrm{~km} \mathrm{~s}^{-1}$, determined in the present paper for 971 RFGC galaxies with $H r<18000 \mathrm{~km} \mathrm{~s}^{-1}$ and $V_{3 \mathrm{~K}}<18000 \mathrm{~km} \mathrm{~s}^{-1}$, is in agreement with the results $V \sim(0-200) \mathrm{km} \mathrm{s}^{-1}$ obtained for the samples EFAR, PSCz, SCI/SCII, as well as SNIa (Riess et al. 1997) 


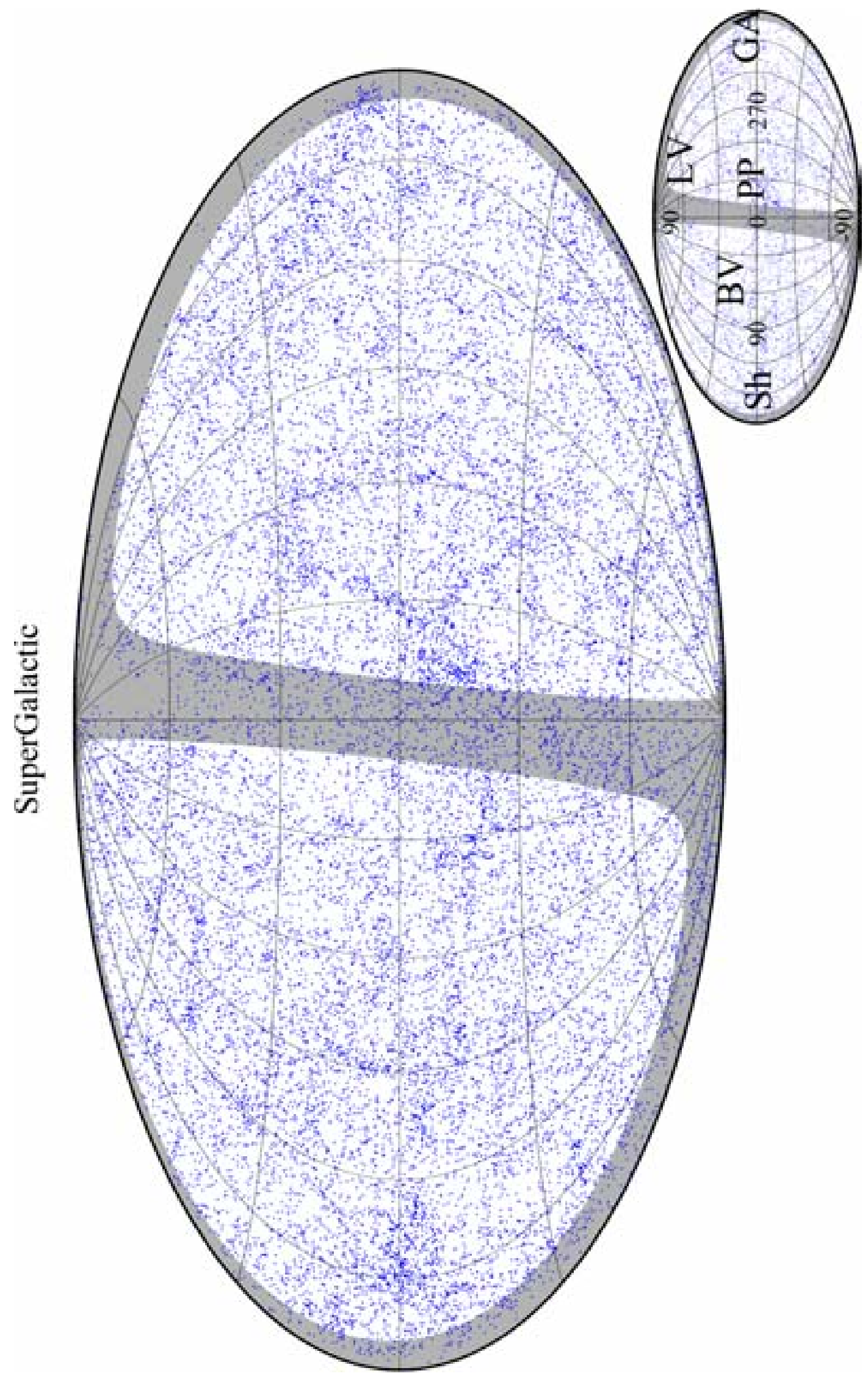

Fig. 5. The sky distribution in $S G L, S G B$ of 22361 galaxies from 2MASS survey with an axial ratio $s b a<0.3$. In the bottom right the positions of the Great Attractor (GA), the Pisces-Perseus (PP), the Shapley concentration (Sh), as well as the Local Void (LV), the Bootes Void (BV) are marked. The "Zone of Avoidance" within Galactic latitude of \pm 10 degrees is shown in grey. 
Table 6. Values of $G$ for several samples of spiral galaxies.

\begin{tabular}{llllll}
\hline \hline Sample & Band & $N$ & $\sigma_{\mathrm{TF}}$ & $G$ & Reference \\
\hline A82 field sample & $H_{-0.5}$ & 359 & 0.47 & 4.0 & Willick et al. (1997) (Mark III) \\
MAT field sample & $I$ & 1355 & 0.43 & 8.6 & Mark III \\
W91PP field sample & $r$ & 326 & 0.38 & 5.0 & Mark III \\
CF field sample & $r$ & 321 & 0.38 & 4.7 & Mark III \\
Great Wall spirals & $I$ & 172 & 0.32 & 4.2 & Dell'Antonio et al. (1996) \\
Spirals in clusters & $I$ & 522 & 0.38 & 6.0 & Dale et al. (1999) \\
Spirals in clusters & $J, H, K_{\mathrm{s}}$ & 153 & 0.48 & 2.6 & Bamford (2002) \\
RFGC & $J, H, K_{\mathrm{s}}$ & 971 & 0.42 & 7.4 & This work \\
\hline
\end{tabular}

which is consistent with the assumption of the flow field convergence to the CMB rest-frame at $100 h^{-1} \mathrm{Mpc}$. However, our result differs strongly from the bulk velocity obtained from the LP10 and LP samples $\left(V \sim 700 \mathrm{~km} \mathrm{~s}^{-1}\right)$. The low bulk velocity at large scales has been predicted by popular theories of structure formation in the cosmological model with cold dark matter and the cosmological constant.

The bulk velocity apex obtained in this paper, $l=301^{\circ} \pm$ $18^{\circ}, b=-2^{\circ} \pm 15^{\circ}$, is located near the apex position $\left(l=282^{\circ}\right.$, $\left.b=-8^{\circ}\right)$ derived from SNIa by Riess et al. (1997) and, within the errors, lies near GA and the massive cluster Abell 3627.

\section{Conclusion}

We used the 2MASS TF relations to obtain parameters of bulk motion for flat edge-on galaxies from RFGC. Because about $71 \%$ of the RFGC sample have $J, H, K_{\mathrm{s}}$ magnitudes from 2MASS, the size of our sample under study is restricted only by the available HI line width data. We have a sample of 1141 all-sky distributed RFGC galaxies at our disposal with both 2MASS magnitudes and velocity/line-width estimates.

After excluding about $15 \%$ of the sample (dwarf galaxies, very distant ones, and also the objects with a deviation over $3 \sigma$ on the TF diagram), we built a set of multi-parametric TF relations using the Kron, isophotal, and extended $J, H, K_{\mathrm{s}}$ magnitudes. The minimal dispersion on the TF diagram is shown to be that for Kron $J_{\text {fe }}$ magnitudes $\left(\sigma_{\mathrm{TF}}=0.422^{\mathrm{m}}\right)$.

For the RFGC sample of $N=971$ galaxies with $V_{3 \mathrm{~K}}<$ $18000 \mathrm{~km} \mathrm{~s}^{-1}$, the bulk velocity and apex position are: $V=$ $199 \pm 61 \mathrm{~km} \mathrm{~s}^{-1}, l=301^{\circ} \pm 18^{\circ}, b=-2^{\circ} \pm 15^{\circ}$. The parameters of bulk motion change insignificantly with the use of other 2MASS magnitudes $J_{\text {20fe }}, H_{20 \mathrm{fe}}, K_{\text {20fe }}, H_{\text {fe }}, K_{\text {fe }}, J_{\text {ext }}, H_{\text {ext }}, K_{\text {ext }}$ ( $V$ changes within $\pm 50 \mathrm{~km} \mathrm{~s}^{-1}, l$ and $b$ within $\pm 15^{\circ}$ ). This result remains robust to a decrease the sample depth. In particular, for $921 \mathrm{RFGC}$ galaxies with $V_{3 \mathrm{~K}}<12000 \mathrm{~km} \mathrm{~s}^{-1}$ we obtained $V=226 \pm 62 \mathrm{~km} \mathrm{~s}^{-1}, l=295^{\circ} \pm 16^{\circ}, b=-2^{\circ} \pm 13^{\circ}$. Within the errors, our estimates of the bulk velocity are in agreement with the data for the EFAR, PSCz, SCI/SCII samples. Furthermore, the apex position and $V$ are consistent with the results obtained for SNIa.

The two-dimensional smoothed peculiar velocity field well traces the large-scale density variations in the galaxy distribution, e.g. Great Attractor, Pisces-Perseus an
Hercules-Coma-Corona Borealis superclusters, Shapley concentration, Bootes and Local voids.

Thus, we show using the RFGC catalogue that the 2MASS Tully-Fisher relation can be used successfully in studying galaxy cosmic flows. The next obvious step is to complete the observations of radial velocities and HI line widths for the remaining RFGC galaxies.

Acknowledgements. We thank Dmitry Makarov for help in the design of Figs. 4 and 5.

This paper makes use of data from the Two Micron All Sky Survey, which is a joint project of the University of Massachusetts and the Infrared Processing and Analysis Center/California Institute of Technology, funded by the National Aeronautics and Space Administration and the National Science Foundation.

We have made use of the LEDA database

( http://leda.univ-lyon1.fr).

This research was partially supported by DFG-RFBR grant 436RUS 113/701/0-1.

\section{References}

Aaronson, M., Huchra, J. P., \& Mould, J. R. 1979, ApJ, 229,1

Aaronson, M., Mould, J. R., \& Huchra, J. P. 1980, ApJ, 237, 655

Aaronsom, M., Huchra, J., Mould, J., et al. 1982, ApJ, 258, 64 [A82]

Bamford, S. P. 2002, Thesis, University of Durham [astro-ph/0210227]

Branchini, E., Teodoro, L., Frenk, C. S., et al. 2000, MNRAS, 308, 1

Colless, M., Saglia, R. P., Burstein, D., et al. 2001, MNRAS, 321, 277 [EFAR]

Cutri, R. M., \& Skrutskie, M. F. 1998, Two Micron All Sky Survey Status Report, BAAS, 30, 1374

Dale, D. A., Giovanelli, R., Haynes, M. P., et al. 1999, AJ, 118, 1489

Dekel, A., Eldar, A., Kollat, T., et al. 1999, ApJ, 522, 1

Dell'Antonio, I. P., Bothun, G. D., \& Geller, M. J. 1996, AJ, 112, 1759 [Great Wall]

Giovanelli, R., Avera, A., \& Karachentsev, I. D. 1997a, AJ, 114, 122

Giovanelli, R., Haynes, M. P., Herter, T., et al. 1997b, AJ, 113, 22

Haynes, M. P., Giovanelli, R., Chamaraux, P., et al. 1999, AJ, 117, 2039

Huchtmeier, W. K., Karachentsev, I. D., Mitronova, S. N., et al. 2003, in preparation

Jarrett, T. H. 2000, PASP, 112, 1008

Jarrett, T. H., Chester, T., Cutri, R., et al. 2000, AJ, 119, 2498

Jarrett, T. H., Chester, T., Cutri, R., et al. 2003, AJ, 125, 525

Karachentsev, I. D., Karachentseva, V. E., Kudrya, Yu. N., et al. 1999, Bull. SAO, 47, 5 [RFGC] 
Karachentsev, I. D., Karachentseva, V. E., Kudrya, Yu. N., et al. 2000a, Bull. SAO, 50, 5

Karachentsev, I. D., Karachentseva, V. E., Kudrya, Yu. N., et al. 2000b, Astron. Zh., 77, 175

Karachentsev, I. D., \& Smirnova, A. A. 2002, Astrofizika, 45, 547

Karachentsev, I. D., Mitronova, S. N., Karachentseva, V. E., Kudrya, Yu. N., \& Jarrett, T. H. 2002, A\&A, 396, 431 (Paper I)

Kilborn, V. A., Webster, R. L., Staveley-Smith, L., et al. 2002, A Catalog of HI-Selected Galaxies from the South Celestial Cap Region of Sky

Kogut, A., Lineweaver, C., Smoot, G. F., et al. 1993, ApJ, 419, 1

Lauer, T. R., \& Postman, M. 1994, ApJ, 425, 418 [LP]

Makarov, D. I., Karachentsev, I. D., Tyurina, N. V., et al. 1997a, Astron. Lett., 23, 445

Makarov, D. I., Karachentsev, I. D., Burenkov, A. N., et al. 1997b, Astron. Lett., 23, 638

Makarov, D. I., Burenkov, A. N., \& Tyurina, N. V. 1999, Astron. Lett., 25,706

Makarov, D. I., Burenkov, A. N., \& Tyurina, N. V. 2001, Astron. Lett., 27,213
Mathewson, D. S., Ford, V. L., \& Buchhorn, M. 1992, ApJS, 81, 413

Mathewson, D. S., \& Ford, V. L. 1996, ApJS, 107, 97

Matthews, L. D., \& van Driel, W. 2000, AAS, 143, 421

Paturel, G., Bottinelli, L., Di Nella, H., et al. 1996, Catalogue of Principal Galaxies, Saint-Genis Laval, Observatoire de Lyon (LEDA)

Riess, A. G., Davis, M., Baker, J., \& Kirshner, R. P. 1997, ApJ, 488, L1 [SNIa]

Saunders, W., Sutherland, W. J., Maddox, S. J., et al. 2000, MNRAS, 317,55

Schlegel, D. J., Finkbeiner, D. P., \& Davis, M. 1998, ApJ, 500, 525

Skrutskie, M. F., Schneider, S. E., Stiening, R., et al. 1997, in The Impact of Large Scale Near-IR Sky Surveys, ed. F. Garzon, et al. (Netherlands: Kluwer), ASSL, 210, 25

Tully, R. B., \& Fouqué, P. 1985, ApJS, 58, 67

Verheijen, M. A. 2001, ApJ, 563, 694

Willick, J. A., Courteau, S., Faber, S. M., et al. 1997, ApJS, 109, 333 [Mark III]

Willick, J. A. 1999, ApJ, 516, 47 [LP10]

Zaroubi, S. 2002 [astro-ph/0206052] 